\title{
Testing the Independent Action Hypothesis of Plant Pathogen Mode of Action: A Simple and Powerful New Approach
}

\author{
Mark P. Zwart and Santiago F. Elena
}

First and second authors: Instituto de Biología Molecular y Celular de Plantas (Consejo Superior de Investigaciones Científicas-Universidad Politécnica de Valencia), 46022 València, Spain; second author: Santa Fe Institute, Santa Fe, NM 87501. Current address of M. P. Zwart: Institute of Theoretical Physics, University of Cologne, 50937 Cologne, Germany. Accepted for publication 26 July 2014.

\begin{abstract}
Zwart, M. P., and Elena, S. F. 2015. Testing the independent action hypothesis of plant pathogen mode of action: A simple and powerful new approach. Phytopathology 105:18-25.

The independent action hypothesis is a simple model of pathogen infection that can make many useful predictions on infection kinetics and, therefore, a number of different tests of independent action have been developed. However, some of these analyses are rather sophisticated, limiting their appeal to experimentalists, and it is also unclear how well the different tests perform. Here, we developed and evaluated a simple and robust new test of independent action. Our new test is based on using

second variant, and then quantifying the infection response for the first variant. We simulated infection data in which we introduced deviations from independent action, experimental variation, or both. Simulations showed that our new procedure has many advantages over the existing tests of independent action, especially if only systemic-infection data are available. We also performed experimental tests of our new procedure using two marked Tobacco etch virus (TEV) variants. We found minor deviations from the independent action model, which were not detected by previous tests using existing methods, exemplifying the utility of this approach. We discuss the implications for TEV infection kinetics and consider how to reconcile different dose-dependent effects.
\end{abstract} a constant inoculum dose of one pathogen variant, varying the dose of a
The independent action hypothesis states that (i) a nonzero probability of infection can be assigned to each pathogen infectious unit and (ii) each infectious unit acts independently of other units $(5,27)$. The hypothesis was - to the best of our knowledgeoriginally formulated and tested for plant viruses. The formation of local lesions (9) makes it relatively straightforward to quantitatively assess the effects of relative dose (as modified by dilution) on infection $(2,12)$. The independent action model not only allows for predictions of the rate of host infection, the number of effectively infecting parasites, and the rate of mixed-variant infections $(25,27)$ but also offers a fundamental description of the most basic interactions that occur when a host organism is exposed to a conspecific pathogen population. Three different approaches have been used to test whether parasites exhibit independent action. Here, we propose a new approach for testing the independent action hypothesis and compare it with existing conventional methods using both simulation and experimental data.

The first conventional approach used for testing for independent action is to consider the relationship between dose and local lesions $(2,7,8,12,16)$. Although this approach is limited to plant viruses and their local lesion hosts, viruses carrying marker proteins can also be used to study the relationship between dose and the number of primary infection foci (25). If there is independent action, then the number of local lesions or primary infection foci $(\lambda)$ should simply be the virion dose $(n)$ times the probability of infection $(p)$. This approach appears to be reasonably robust a priori, because low levels of experimental variation (i.e., differences in host susceptibility or administered dose, affecting $p$ and $n$ values, respectively) will not affect the mean of $\lambda$ but only increase its variation. For higher doses, the number of local lesions

Corresponding author: Mark P. Zwart; E-mail address: mzwart@uni-koeln.de

http://dx.doi.org/10.1094/PHYTO-04-14-0111-R

(c) 2015 The American Phytopathological Society tends to saturate, due to the inability to distinguish individual local lesions or a limited number of infection sites (8). On the other hand, this approach has proven to be useful, because deviations from independent action predictions led to the discovery of multipartite viruses $(7,16)$.

A second approach to test for independent action has been to consider the relationship between dose and response (i.e., infection status: the binomial "noninfected" or "infected"). This approach, originally proposed by Druett (5) to describe infection kinetics for anthrax spores, has been refined in many subsequent studies $(18,22)$. The underlying principle is that independent action leads to a dose response with a fixed, sigmoidal shape (18) given as $I=1-e^{-p n}$, where $I$ is the fraction of infected hosts and $e^{-p n}$ is the zero term for a Poisson-distributed number of founders (i.e., those that are not infected). When modeling infection, we assume that primary infection invariably leads to the establishment of systemic infection, as is the case for our experimental models system (25), and, therefore, $p$ is the infection probability for both primary and systemic infection. One important limitation to this approach for testing independent action is that both antagonism between individual parasites, variation in the actual dose administered, and heterogeneity in the host susceptibility to the parasite can lead to a shallower dose response $(3,18,19,24)$. Therefore, deviations from the simple independent action model with a fixed probability of infection can occur for a number of reasons, and may not be indicative of dose-dependent interactions in the parasite population (24).

A third approach to test for independent action has been to consider the frequency of mixed-variant infections, when host organisms are challenged with an inoculum composed of two or more pathogen variants. One can first estimate $\lambda$-assuming the number of infecting parasites follows a Poisson distribution-from the fraction of uninfected hosts (27) as $\lambda=-\ln (1-I)$ and, given the frequency of one of the variants in the inoculum $\left(f_{A}=1-f_{B}\right)$, it follows that $\operatorname{Pr}(A \cap B)=\operatorname{Pr}(A) \times \operatorname{Pr}(B)=\left(1-e^{-f_{A} \lambda}\right)\left(1-e^{-\left(1-f_{A}\right) \lambda}\right)$. 
Therefore, the level of infection and frequency of variants in the inoculum can be used to predict the level of mixed-variant infections. This approach has also been used qualitatively, based on the idea that a small number of infecting pathogens should lead to infection mainly by a single pathogen variant, whereas a large number of infecting pathogens should lead to many mixed infections $(1,15,21)$. This approach is probably the least useful for testing for independent action, because experimental variation will lead to higher levels of mixed-variant infection (24). However, it has been suggested that this approach may be used in combination with others to test for independent action $(24,27)$.

Although multiple tests for independent action have been proposed, there are many caveats with these tests that lead to complications with their interpretation. This, in turn, requires rather sophisticated analysis with somewhat counterintuitive results (3, 24), which experimentalists may not be keen to employ. Therefore, here, we set out to develop an alternative test of independent action which was (i) highly robust and not subject to limitations of other tests and (ii) parsimonious in setup and analysis, requiring only everyday statistical tests and allowing for an intuitive explanation of the results. We first evaluated the new tests of independent action developed here with simulation data, allowing consideration of test results under a wide range of conditions. We then performed experiments using this new approach with Tobacco etch virus (TEV; genus Potyvirus, family Potyviridae) and Nicotiana tabacum L. 'Xanthi' plants. Because we had already performed the existing tests of independent action with this model system, we could then contrast the results of the new and old tests.

\section{MATERIALS AND METHODS}

New testing procedure. Whereas previous tests of independent action always vary the dose of both virus variants, the key difference with the new testing approach we are proposing is that the dose of one virus variant $(A)$ is held constant, while the dose of the other virus variant $(B)$ is varied (Fig. 1). Subsequent statistical tests then determine whether the infection response (i.e., the number of primary foci or the frequency of mixedvariant infections) is the same for $A$ when the dose of $B$ is varied. There are two advantages to holding the dose of one of the virus variants constant: (i) it simplifies testing, because the statistical procedure only needs to test for an effect of the dose of variant $B$ on the infection response for variant $A$, and (ii) it should make the test more sensitive, because the new test can compare the infection response of variant $A$ with any range of dose values for variant $B$.

In more detail, the following setup and analysis are proposed as an alternative test for independent action. Two conspecific virus variants, differing only in a marker sequence or protein, are used to make a series of mixtures, in which the dose of one virus variant is varied while the other is held constant (e.g., for the $j$ th dose $n_{A}=10^{c}$ and $n_{B}=10^{c+j-1}$, where $c$ is a dose resulting in an appropriate level of infection). Subsequently, there are two possible ways to further analyze the data. First, if possible. the number of primary infection foci of variant $A$ are quantified; then, a nonparametric procedure is used to determine whether the dose of variant $B$ has an effect on the number of foci of variant $A$. For example, Spearman's rank correlation can be calculated for $n_{B}$ and $\lambda_{A}$ data. In this case, the value of $c$ ideally should be high enough that all hosts are infected with variant $A$, although the exact infection level does not materially impact the test. Second, if foci of primary infection cannot be quantified but the infection status can be determined for the two variants (e.g., by means of polymerase chain reaction [PCR] or the presence of a marker protein), then a nonparametric procedure can be used to test whether dose of variant $B$ has an effect on the frequency of hosts infected by variant $A$. For example, a trend test for equal propor-

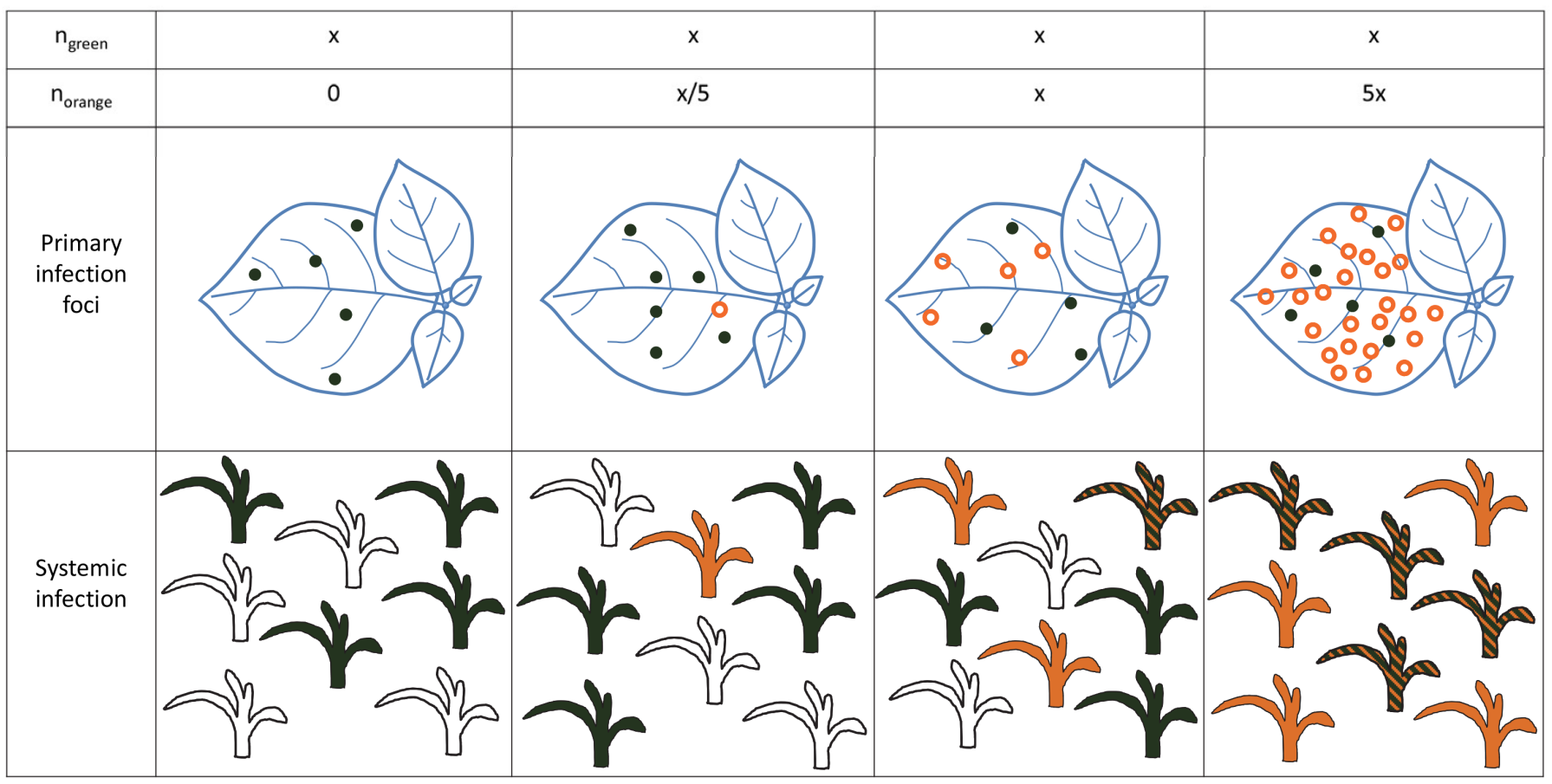

Fig. 1. This figure conceptually illustrates the approach we propose here for testing independent action. The dose of one virus variant ( $\left.\mathrm{n}_{\text {green }}\right)$ is held constant, while the dose of a second variant $\left(\mathrm{n}_{\text {orange }}\right)$ is varied. If there is independent action, the response of the green variant should be constant despite the changes in dose of the orange variant. The response that is analyzed can be primary infection foci (illustrated with plant leaves), in which case the number of foci of the green variant (open circles) should stay the same despite increases in the number of foci of the orange variant (closed circles). The analyzed response can also be systemic infection, as illustrated with the plants at the bottom of the figure. In this case, no fill indicates uninfected hosts while diagonal stripes indicate mixedvariant systemic infections. If there is independent action, then the frequency with which one virus variant systemically infects plants should be independent of the dose (and frequency of infection) of the other variant. Note that systemic infection frequency for the variant kept at constant dose includes both single-variant "green" infections and mixed-variant infections. 
tions can be used. In this case, $c$ should be chosen so that approximately half of the hosts are infected with variant $A$ (in the absence of variant $B$ ). This will allow the frequency to increase or decrease, should there be synergistic or antagonistic dose-dependent effects. A nonparametric analysis of variance could also be used as the statistical test in both cases. The advantage of the statistical procedures suggested-Spearman's rank correlation or a trend test of equal proportions-is that they use information on the order of doses and two specific hypotheses (decrease or increase of number or frequency with dose) and, thereby, will increase statistical power for the detection of dose-dependent effects. At the same time, when combined with the approach we are proposing, no assumptions need to be made on deviations from model predictions, other than that possible dose-dependent effects are monotonic.

Generation of simulation data. As a first test of whether the new test of independent action we have suggested is superior to conventional tests, we simulated the infection of hosts with two conspecific virus variants and then performed all five tests, using R 3.0.2 (17). Our simulations consisted of assuming a Poissondistributed number of infecting virus particles for two virus variants (with means $\lambda_{A}$ and $\lambda_{B}$ ), with the same probability of infection $(p)$ and same frequency in the inoculum $\left(f_{A}=f_{B}=0.5\right.$ for all simulations). From these simulated data, we then determined how many foci of primary infection were present (equal to the total number of infecting virus particles), the frequency of infected hosts $I$, and the frequency of hosts infected by one variant, $f(A)$ and $f(B)$, or both $f(A \cap B)$.

To generate data for which independent action did or did not hold (the so called "dependent action" model), we used the infection model $\lambda=p n^{\mathrm{K}}(18)\left(n=n_{A}+n_{B}\right)$, and then partitioned $\lambda$ over the two virus variants (i.e., $\lambda_{A}=f_{A} \lambda=$ and $\lambda_{B}=f_{B} \lambda$ ). The constant $\kappa$ determines the interactions between virus particles during infection. If $\kappa=1$, there is independent action, whereas when $\kappa \neq 1$, there is some form of dose-dependent action. When $\kappa<1$, there is antagonistic dependent action (the probability of infection per virus particle decreases as more virus particles are introduced), whereas when $\kappa>1$, there is synergistic dependent action (the probability of infection increases as more virus particles are introduced). In the simulations, we used $\kappa$ values of $10^{-0.3}, 10^{-0.2}, 10^{-0.1}, 10^{-0.05}, 10^{0}, 10^{0.05}, 10^{0.1}, 10^{0.2}$, and $10^{0.3}$, thereby focusing on relatively small deviations from the independent action model. Additionally, we varied the amount of experimental variation in the simulated data, by multiplying $p$ with a value drawn from a $\beta$ distribution (with shape parameters $\alpha$ and $\beta$ ) in effect allowing host susceptibility to the virus to follow a $\beta$ distribution over hosts. The $\beta$ distribution was chosen for versatility, in particular to allow for bimodal distributions of host susceptibility. For all simulations, $\alpha=\beta$ and, therefore, the mean of the $\beta$ distribution is constant while the variation of the distribution decreases with increasingly high $\alpha$ and $\beta$ values. We used $\alpha=\beta$ values of $10^{-4}, 2,12,62$, and 312 , resulting in variances 0.25 , $0.05,0.01,2 \times 10^{-3}$, and $4 \times 10^{-4}$, respectively, of the $\beta$ distribution for host susceptibility. The distribution is consequently bimodal for the highest variance and unimodal for all lower variances, $p$ is the highest probability of infection value that can be drawn, and the mean of the distribution of susceptibilities is $p / 2$. Therefore, we simulated both deviations from the independent action, heterogeneity in host susceptibility, or both, rendering data over a range of conditions for which the conventional tests may fail (24).

For each combination of $\kappa$ and $\alpha=\beta$ parameter values, we simulated seven doses and 15 replicate hosts per dose. We set $p=$ 0.001 and adjusted the dose range and intervals between doses for each different test of independent action, so that the doses would result in a suitable response range in which deviations from model predictions might be detected. We also adjusted the $p$ value by exponentiation with $\kappa$, so that when $\kappa \neq 1$, the dose would still be in a suitable range. This adjustment is equivalent to having prior information from, for example, a pilot experiment on what dose range to use and, therefore, was compatible with our aims. For the conventional tests of independent action, we used the following total doses of virus particles, composed of equal fractions of the two variants: (i) for the dose versus response, dose versus mixedvariant infection simulations, and dose primary infection foci, doses were $10^{1.5}, 10^{2}, 10^{2.5}, \ldots, 10^{4.5}$ virus particles; and (ii) for the dose versus primary infection foci simulations, we also used a range of higher doses: $10^{3}, 10^{2.5}, 10^{4}, \ldots, 10^{6}$ virus particles. For the simulations for the new tests of independent action, we used doses of $0,10^{1}, 10^{2}, \ldots, 10^{6}$ of variant $A$ virus particles and a fixed dose of $10^{3}$ virus particles of variant $B$.

Performing model tests on simulated data. To perform tests of independent action on the simulated data, we used the following procedures. For dose response data, we fitted both the independent action and the dependent action $\left(\lambda=p n^{\kappa}\right)$ models to the data using grid searches over a large parameter space. The best solution for the grid space was determined by considering the negative log-likelihood, obtained from the binomial likelihood of model predictions:

$$
L\left(I_{j} \mid W_{j}, V_{j}\right)=\left(\begin{array}{c}
W_{j} \\
V_{j}
\end{array}\right) I_{j}^{W_{j}}\left(1-I_{j}\right)^{W_{j}-V_{j}}
$$

where $W$ is the total number of inoculated hosts (15) and $V$ is the number of infected hosts. The sum of the negative log-likelihood for all doses was used to determine the Akaike information criterion, and we used the Akaike Weight (AW) to determine whether one model was supported (with a threshold value of 0.05 ).

For dose primary-infection-foci data, we fitted the independent action and the dependent action models to the $\log _{10}$-transformed data with grid searches, calculated the likelihood value corresponding to the residual sum of squares (10), and then also used the AW to determine which model was better supported.

For mixed-variant infections, we simply used a binomial test to compare model predictions for the level of mixed infection based on the level of noninfection to observed values. Because this led to multiple test values, we then used Fisher's method to obtain a single $P$ value, and determined whether it was lower than the threshold value of 0.05 .

To perform the new tests of independent action on the simulated data, we used Spearman's rank correlation to test whether the number of foci of variant $A$ changed with the dose of variant $B$. A trend test of equal proportions was used to evaluate whether the frequency of hosts infected with variant $A$ changed with the dose of variant $B$.

Experiments with TEV and tobacco plants. The two virus variants used in our experiments were TEV-enhanced green fluorescent protein (eGFP) and TEV-mCherry (25), which express the eGFP or the red fluorescence protein mCherry. These viruses have similar infectivity and fitness, and the marker sequences are very stable during short-duration infection $(25,28)$. As an inoculum, we used symptomatic tissue from plants infected individually with each virus, collected at 10 days postinoculation (dpi). The tissue was ground to a fine powder, RNA was extracted, and a quantitative real-time reverse-transcription PCR, with specific primers for the TEV coat protein cistron, was performed to determine virus accumulation (28). Ground tissue of both viruses was then resuspended in inoculation buffer $(50 \mathrm{mM}$ potassium phosphate $[\mathrm{pH} 7.0]$ and $3 \%$ polyethylene glycol 6000), and mixtures of both virus variants were made in inoculation buffer. In these inoculum mixtures, the dose of TEV-eGFP was held constant $\left(5.34 \times 10^{6}\right.$ virions per $\left.5 \mu \mathrm{l}\right)$ while the dose of TEVmCherry was varied $\left(0,1.78 \times 10^{6}, 5.34 \times 10^{6}, 1.60 \times 10^{7}\right.$, or $4.81 \times 10^{7}$ virions per $\left.5 \mu \mathrm{l}\right)$. Plants were rub inoculated with $5 \mu \mathrm{l}$ of these mixtures and $2 \mu \mathrm{l}$ of $10 \%$ carborundum, and primary infection foci were quantified 3 dpi. We quantified TEV-eGFP foci and TEV-mCherry primary infection foci, and also carefully 
noted the number of mixed foci: those foci which gave both eGFP and mCherry fluorescence. Two experiments were performed: the first one with 21-day-old $N$. tabacum plants and the second one with 28-day-old $N$. tabacum plants. The older plants would be expected to be somewhat more resistant to virus infection and, as a result, we expected differences in the mean number of primary infection foci for a given dose.

To estimate $\kappa$ (the constant that determines interactions between virions during the infection process) for the TEV-eGFP foci data, we separately fit the dependent action infection model to data from both experiments. The number of TEV-eGFP foci was predicted to be $\lambda_{e G F P}=f_{e G F P} p\left(n_{e G F P}+n_{m C h e r r y}\right)^{\mathrm{K}}$, and the residual sum of squares was minimized by means of a grid search over large parameter spaces of $p$ and $\kappa$.

\section{RESULTS AND DISCUSSION}

Results of conventional tests with simulation data. An ideal test of independent action would (i) tend to give a significant result if $\kappa$ deviates slightly from 1 and (ii) be insensitive to changes in experimental variation, giving neither false positive or negative results (Fig. 2A). An evaluation of the conventional tests of independent action on simulated data shows that the tests all have some important limitations (Fig. 2B and E). The dose primaryinfection-foci test is probably the best of the three conventional tests (Fig. 2B and C) because, with a high dose range, it is sensitive to departures from independent action, and low to intermediate levels of variation in host susceptibility do not affect the test results. However, for high levels of variation in host susceptibility, the test gives false-negative results for values of $\kappa<1$ and false positives for $\kappa=1$. Moreover, using high dose values $\left(10^{3}\right.$ to $10^{6}$ ) is probably not very relevant from an experimental perspective, because the resulting numbers of primary infection foci or local lesions could not be counted in an actual experiment (25), leading to saturation in the observed number of foci or lesions. Therefore, we repeated the analysis for lower dose values $\left(10^{1.5}\right.$ to $10^{4.5}$ ), which lowered the sensitivity of the test (Fig. 2C) while also lessening the false positives under high variation in host susceptibility. False negatives when $\kappa<1$ remained at high levels of variation in host susceptibility. In summary, the dose primaryinfection-foci test has some limitations but is a reasonable test of independent action. This result is in good agreement with early use of this test which, for example, led to the discovery of multipartite plant viruses (7). The primary limitation of this test is that it can only be used in those host-virus pathosystems where the primary infection sites could actually be quantified.

The dose-response test for independent action performed rather poorly with the simulated data (Fig. 2D). The test was not very sensitive to deviations from independent action, especially when $\kappa>1$, and gave false-positive results with high levels of variation in host susceptibility. Ben-Ami et al. (3), Regoes et al. (18), and Ridout et al. (19) have previously highlighted limitations of the dose response test. The results presented here further reinforce the notion that the most commonly used procedure for testing whether independent action holds is, in fact, a poor test.

The mixed-variant infection test gave even poorer results (Fig. 2E) than the dose-response test (Fig. 2D). This procedure in and of itself really only tests whether there is variation in host susceptibility, and even then only gives a significant outcome for high levels of variation in host susceptibility. This setup of using two marked variants is valuable for estimating the number of founders of infection (20). However, as suggested by van der Werf et al. (24), this procedure itself does not give insight into whether independent action applies to a particular host-virus pathosystem.

Therefore, performing the conventional tests of independent action on simulation data gives disparate results. The relationship between dose and the number of primary infection foci is the most informative test, although it does have some limitations when variation in host susceptibility is high. On the other hand, tests based on dose response and mixed-variant infections are severely limited, often rendering false-positive and false-negative results. Therefore, our simulation results strongly highlight the limitations of these individual tests, hereby lending support to the view that only a combined analysis of dose response and mixedvariant infection (24) provides insight into whether independent action holds. Such a combined analysis provides insight because considering mixed-variant infections identifies the level of variation in host susceptibility, allowing one to-in essence-test whether the shape of the predicted independent action dose response corresponding with this level of variation matches experimental data. However, without such an elaborate analysis, both the dose-response and mixed-variant infection tests are of limited value for testing independent action.

Results of new tests of independent action proposed here. The new tests of independent action proposed here were generally superior to the conventional tests. The procedure we propose to test independent action for dose versus primary infection foci (Fig. 2F) is more sensitive than the conventional test on low dose data (Fig. 2C). Moreover, variation in host susceptibility did not lead to false positives for our procedure while at the same time leading only to a marginal increase in false negatives for small deviations from independent action predictions (Fig. 2F). Note that our procedure allows an extreme dose range to be used for the virus variant for which dose is varied; only the primary infection foci for the variant for which dose is held constant actually need to be quantified to permit a test. The usable dose range is probably one of the reasons why the procedure we describe here has a high sensitivity to departures from independent action.

The new testing procedure for mixed-variant infections (Fig. $2 \mathrm{G}$ ) is markedly superior to the dose-response or mixed-variant infection tests. This new procedure should be contrasted with these two conventional procedures, because all these procedures do not rely on primary infection foci and, therefore, can be broadly applied in any pathosystem where systemically infecting virus variants can be detected. The test was less sensitive to small deviations from independent action than the procedures for dose versus primary infection foci but still reasonably sensitive. Moreover, the test only loses sensitivity for detecting deviations from independent action under one combination of circumstances: (i) the highest levels of variation in host susceptibility and (ii) $\kappa<1$. Given that the conventional tests of independent action are based on frequency of infection and mixed-variant infection, it can be stated that the test we propose represents a marked improvement. Therefore, an evaluation of the alternative tests of independent action with simulated data suggests that our new tests are both superior to the conventional tests of independent action, being more likely to detect deviations from independent action and less likely to give false-positive results due to host variation.

Evaluation of new tests of independent action for TEV. We then considered how well our new testing procedure performs on experimental data. We chose TEV and $N$. tabacum as a model pathosystem, because it has previously been shown that the conventional tests of independent action considering dose-response and mixed-variant infection found no significant deviations from independent action for this system (25). The dose-foci relationship also does not differ significantly from independent action for low doses (25) whereas, at high doses, it was previously found that the relationship appears to attenuate, and $\kappa<1$ was estimated (14). Therefore, we would expect a priori to either (i) confirm support for the independent action model or (ii) find small deviations from the independent action model, which would not be detected by the less-sensitive conventional tests of independent action.

For our experiments, tobacco plants were infected with two TEV variants with stable fluorescent markers (25) eGFP or 

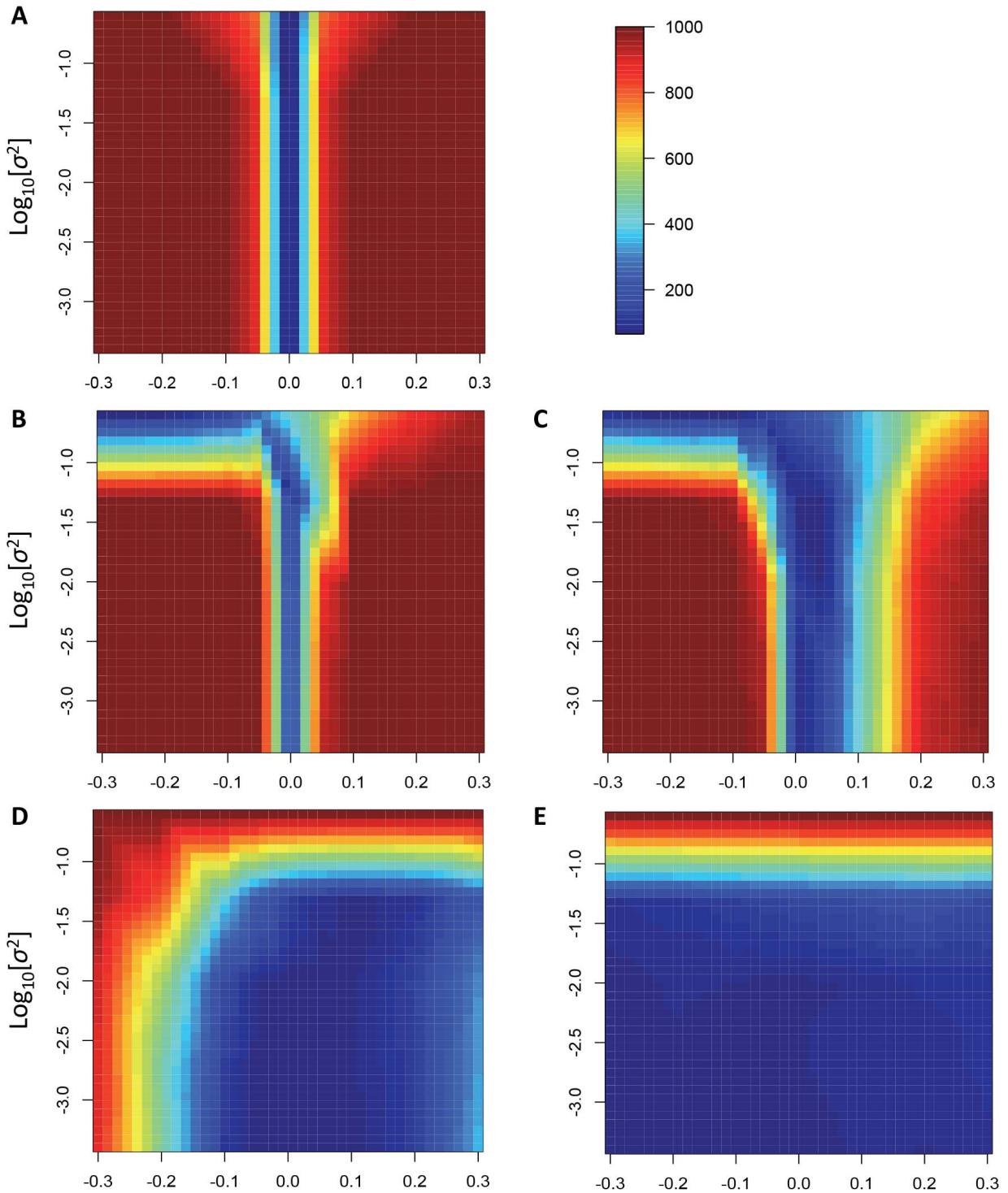

E
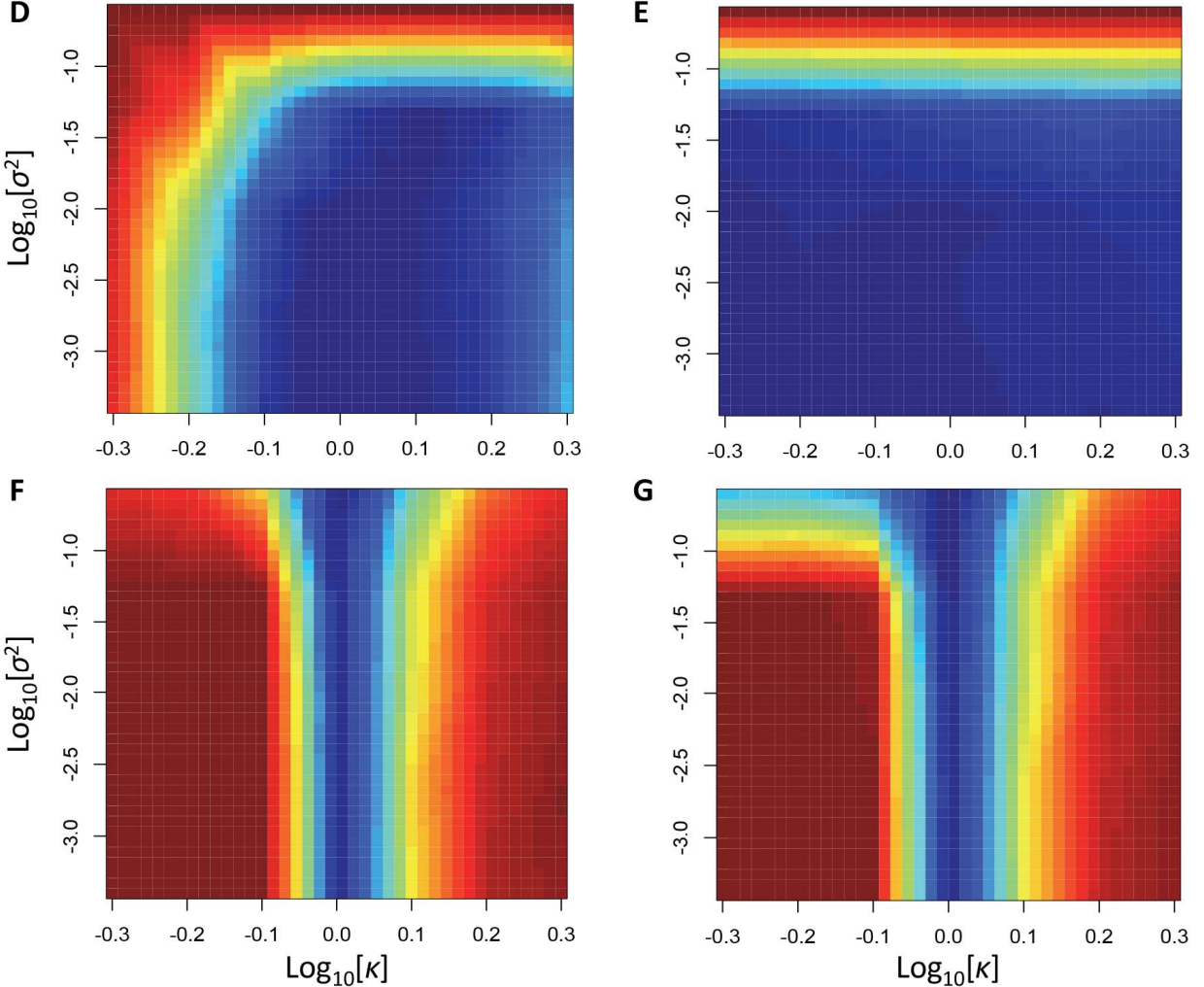

G

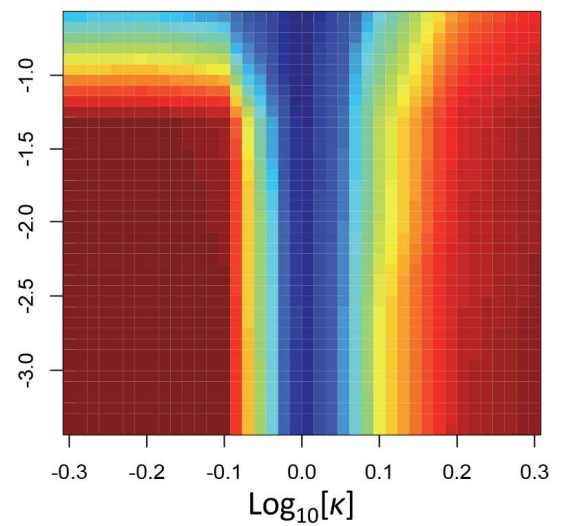

Fig. 2. Results of the conventional and new approaches to testing independent action for simulated data. For all panels, the abscissa is the $\log _{10}$-transformed $\kappa$ value, while the ordinate is the $\log _{10}$-transformed variance of the $\beta$-distributed host susceptibility. The heat maps indicate the number of statistically significant results for 1,000 simulated data sets, as indicated in the legend in the top right corner. A, We propose what the results would look like for an ideal test of independent action: the test would detect small deviations from independent action $\left(\log _{10} \kappa \neq 0\right.$ ), while increases in variation in host susceptibility would not materially affect the test outcome. B, Results of the test based on primary infection foci. In this panel, the dose range is probably too high to be very meaningful to real experimental systems. C, Results of the test based on primary infection foci for lower, more realistic doses. In B and C, the test performs reasonably well, although high levels of variation do result in false-negative results when $\log _{10} \kappa<0$. D, Results for the dose-response test of independent action, which performs poorly. The test is not sensitive to small deviations from independent action, and always gives false positives when there is high variation in host susceptibility. E, Results of the test for independent action based on the frequency of mixed-variant infection. The results clearly show that this test, on its own, is not useful for determining whether independent action holds or not. F, Results for our new procedure for testing independent action based on primary infection foci. The test performs well, and is least affected by high levels of variation in host susceptibility. G, Results for the new procedure based on mixed-variant infection. This procedure should be compared to D (dose response) and $\mathrm{E}$ (frequency of mixed-variant infection), because this test does not require information on the number of primary infection foci. This test is markedly superior to the two conventional tests based on frequency of systemic infection. 

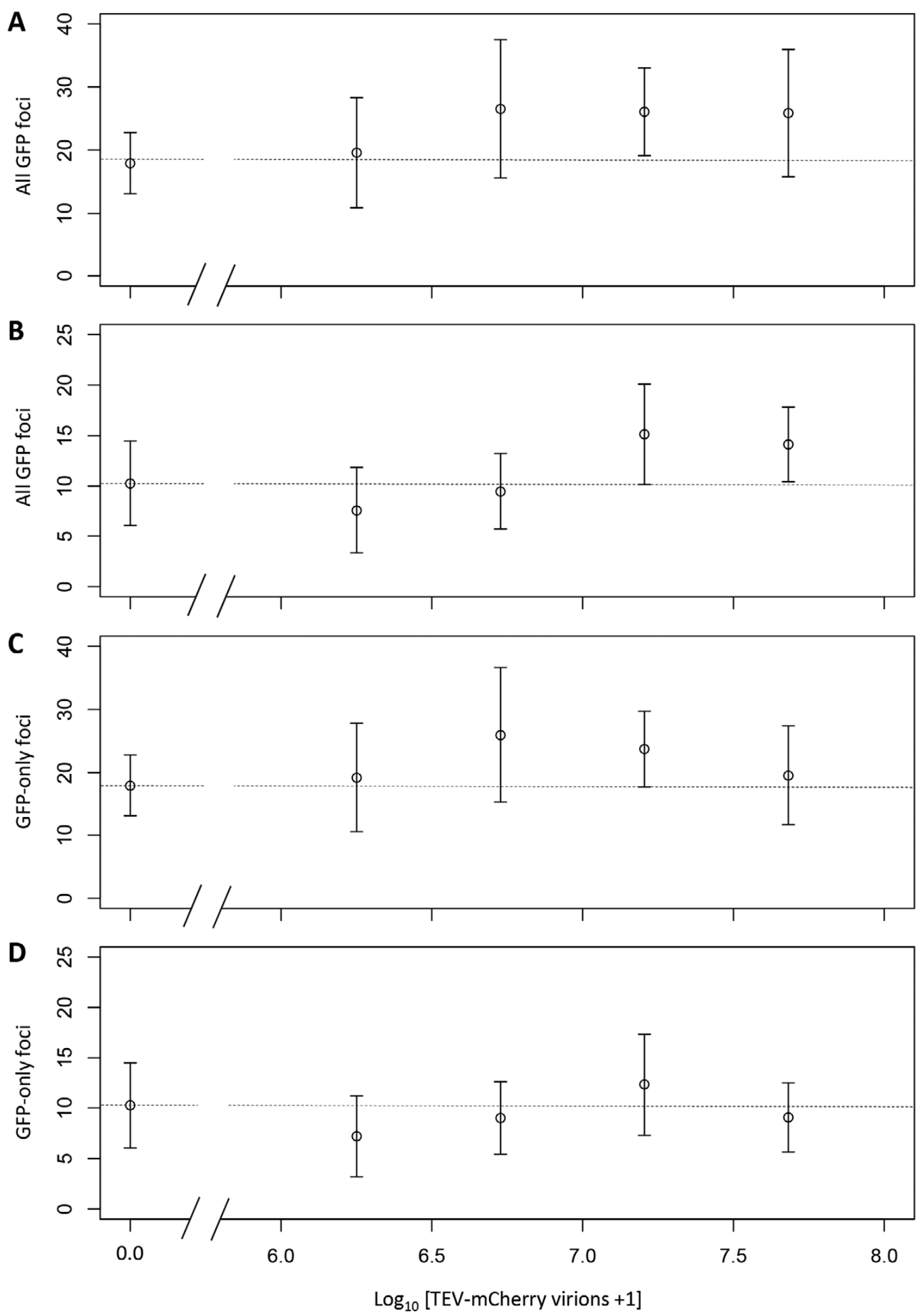

Fig. 3. For all panels, the $\log _{10}$-transformed dose of Tobacco etch virus (TEV)-mCherry is on the abscissa and the number of TEV-enhanced green fluorescent protein (eGFP) foci is on the ordinate. Note that, whereas the TEV-mCherry dose is increased, the dose of TEV-eGFP is held constant over all inoculum mixtures and, according to the null hypothesis (independent action), the number of TEV-eGFP foci should not change as the dose of TEV-mCherry is increased. A and B, Total number of foci that showed eGFP expression, including mixed-variant foci that also showed mCherry expression. C and D, Number of foci that showed only eGFP expression. A and C, Results for experiment 1 (21-day-old N. tabacum plants) and B and D, results for experiment 2 (28-day-old $N$. tabacum plants). When the total number of foci is considered (A and B), there was a small but highly significant increase in TEV-eGFP foci number as the dose of TEV-mCherry increased for both experiments. This increase indicates a synergistic dose-dependent interaction. When only single-variant eGFP foci are considered (C and D), there were no significant dose-dependent effects. Hence, the observed effect appears to be dependent on the formation of mixed-variant foci. 
mCherry. We found a small increase in the total number of primary infection foci of TEV-eGFP (held at constant dose) as the dose of TEV-mCherry was increased (Fig. 3A and B). However, this effect was highly significant in both experiments (Spearman's correlation; experiment 1: $\rho=0.383,73 \mathrm{df}, P<0.001$; experiment 2: $\rho=0.424,73 \mathrm{df}, P<0.001)$. The number of TEV-eGFP foci increased as the dose of TEV-mCherry increased, suggesting that there are small synergistic effects between TEV virus particles during the infection process. To consider what mechanisms might underlie this observation, we then performed the same analysis on the number of primary infection foci for which we observed only eGFP and no mCherry signal; in other words, those foci in which only the TEV-eGFP virus appears to be present (Fig. 3C and D). In this case, we found no statistically significant effects of the dose of TEV-mCherry on the number of TEV-eGFP foci (Spearman's correlation; experiment 1: $\rho=0.147$, $73 \mathrm{df}, P=0.210$; experiment 2: $\rho=0.071,73 \mathrm{df}, P=0.543$ ). Therefore, the deviations from independent action model predictions are apparently due to the occurrence of mixed-variant primary infection foci, suggesting that the establishment of a primary infection focus by one variant allows the other variant to infect more easily. This result is surprising, given that plant viruses often exhibit exclusion $(4,6,11,13)$. However, for TEV, it has been shown that virus variants do not exclude each other at the cellular level following concurrent inoculation (23), making this finding more plausible and consistent with TEV infection dynamics.

From the data of both experiments with TEV, we used the dependent action model to estimate that $\kappa$ ( \pm standard error of the mean) was $1.152 \pm 0.042$, a value that is significantly $>1(z=$ $3.619, P<0.001$ ) (see "Experiments with TEV and tobacco plants" in the Materials and Methods section for details), indicating that the observed synergistic effect was weak. The number of TEV-eGFP foci appears to saturate rapidly as TEV-mCherry dose is increased in experiment 1 (Fig. 3A), suggesting that this synergistic effect reaches its maximum strength very quickly. This idea is congruent with previous results, were it was found that, even at extremely high doses, the frequency of TEV mixed-variant foci always remains low (25).

Conceptual model of TEV infection kinetics. For TEV, results have been produced that are compatible with all infection models considered here. First, independent action was supported by studies in which TEV was inoculated at low doses $(25,26)$. On the other hand, Lafforgue et al. (14) observed that the number of primary infection foci increases disproportionately less than expected for higher doses. This observation may be similar to the saturation of the number of local lesions reported in classical plant virus studies (8). Finally, here, we found evidence for weak synergistic dose-dependent effects. How can these paradoxical results be explained?

The difference between finding independent action or antagonistic dependent action for a test considering the relationship between dose and number of foci appears to depend on the dose range; the number of primary infection foci is similar to independent action model predictions for the low dose range in the study of Lafforgue et al. (14), whereas our other studies did not consider very high doses $(25,26)$. This antagonistic effect at high doses could occur for a number of reasons, including a limited number of infection sites or the inability to discriminate individual infection sites at higher doses (8).

For our new test procedure, we only found significant differences when mixed-variant foci were considered, whereas the independent action model was not rejected when mixed-variant foci were excluded from the analysis. Therefore, the three results are reconcilable when considering the number of foci present in the inoculated leaf, because the synergistic effect we show appears to affect the type but not the total number of foci. Therefore, when mixed-variant foci are included in the analysis, our new approach tests for dose independence in a different infection attribute from that being tested in procedures considering the total number of foci, which do not incorporate qualitative information on foci. This result emphasizes that, in practice, it will not always be possible to compare our new approach one-to-one with existing approaches in all cases.

The synergistic effects we found only result in a small increase in the number of mixed-variant foci in the inoculated leaf, meaning that the size of the virus-population-founding infection will be larger, while the number of foci observed stays the same. Although this effect was observed for an intermediate number of foci (10 to 100 foci), mixed-variant primary infection foci are still scarce at extremely high doses (25). These observations suggest that this effect is never very strong and is already maximized at intermediate doses and, as such, it is of minor importance for describing potyvirus infection kinetics. A mechanistic basis for the synergistic effect we have observed may be that only a limited number of sites that can be effectively infected by two or more virions and, therefore, can display mixed-variant infection.

Concluding remarks. We conclude that our new experimental design for testing independent action appears to have advantages over the conventional approaches described to date. For simulation data, this approach proved to be sensitive to small deviations from independent action, while increases in experimental variation led to only small reductions in power but never resulted in the unwarranted rejection of independent action. When our new approach was tested experimentally with TEV, we found a small synergistic deviation from TEV that had not been previously detected using conventional approaches. This deviation is of a small magnitude and, therefore, will have limited biological implications, and only appears to qualitatively affect foci formation. Consequently, our results here do support the notion that the independent action model does give a reasonable approximation of TEV infection kinetics, in particular at low doses. On the other hand, the fact that our new approach detected these small deviations from independent action reinforces the idea that these new procedures are superior to the conventional tests of independent action.

\section{ACKNOWLEDGMENTS}

We thank F. de la Iglesia and J. Forment for excellent technical support and the IBMCP Bioinformatics Core Service for computational support. This work was supported by grant BFU2012-30805 (to S. F. Elena) and by Juan de la Cierva postdoctoral contract JCI-2011-10379 (to M. P. Zwart) from the Spanish Ministry of Economy and Competitiveness.

\section{LITERATURE CITED}

1. Ali, A., Li, H., Schneider, W. L., Sherman, D. J., Gray, S., Smith, D., and Roossinck, M. J. 2006. Analysis of genetic bottlenecks during horizontal transmission of Cucumber mosaic virus. J. Virol. 80:8345-8350.

2. Bald, J. G. 1937. The use of numbers of infections for comparing the concentration of plant virus suspensions I. Dilution experiments with purified suspensions. Ann. Appl. Biol. 24:33-55.

3. Ben-Ami, F., Regoes, R. R., and Ebert, D. 2008. A quantitative test of the relationship between parasite dose and infection probability across different host-parasite combinations. Proc. R. Soc. B 275:853-859.

4. Dietrich, C., and Maiss, E. 2003. Fluorescent labelling reveals spatial separation of Potyvirus populations in mixed infected Nicotiana benthamiana plants. J. Gen. Virol. 84:2871-2876.

5. Druett, H. A. 1952. Bacterial invasion. Nature 170:288.

6. Folimonova, S. Y. 2012. Superinfection exclusion is an active viruscontrolled function that requires a specific protein. J. Virol. 86:5554-5561.

7. Fulton, R. W. 1962. Effect of dilution on Necrotic ringspot virus infectivity and enhancement of infectivity by noninfective virus. Virology $18: 477-485$

8. Furumoto, W. A., and Mickey, R. 1967. A mathematical model for infectivity-dilution curve of Tobacco mosaic virus-experimental tests. Virology 32:224-233.

9. Holmes, F. O. 1929 Local lesions in tobacco mosaic. Bot. Gaz. 87:39-70.

10. Johnson, J. B., and Omland, K. S. 2004. Model selection in ecology and evolution. Trends Ecol. Evol. 19:101-108. 
11. Kell, A. M., Wargo, A. R., and Kurath, G. 2013. The role of virulence in in vivo superinfection fitness of the vertebrate RNA virus Infectious hematopoietic necrosis virus. J. Virol. 87:8145-57.

12. Kleckowski, A. 1950. Interpreting relationships between concentrations of plant virus and numbers of lesions. J. Gen. Microbiol. 4:53-69.

13. Kunkel, L. O. 1934. Studies on acquired immunity with tobacco and aucuba mosaic. Phytopathology 24:437-466.

14. Lafforgue, G., Tromas, N., Elena, S. F., and Zwart, M. P. 2012. Dynamics of the establishment of systemic Potyvirus infection: Independent yet cumulative action of primary infection sites. J. Virol. 86:12912-12922.

15. Moxon, E. R., and Murphy, P. A. 1978. Hemophilus influenzae bacteremia and meningitis resulting from survival of a single organism. Proc. Natl. Acad. Sci. USA 75:1534-1536.

16. Price, W. C., and Spencer, E. L. 1943. Accuracy of the local lesion method for measuring virus activity. II. tobacco necrosis, alfalfa mosaic, and tobacco ringspot viruses. Am. J. Bot. 30:340-346.

17. R Development Core Team. 2013. R: A Language and Environment for Statistical Computing. R Foundation for Statistical Computing, Vienna.

18. Regoes, R. R., Hottinger, J. W., Sygnarski, L., and Ebert, D. 2003. The infection rate of Daphnia magna by Pasteuria ramosa conforms with the mass-action principle. Epidemiol. Infect. 131:957-966.

19. Ridout, M. S., Fenlon, J. S., and Hughes, P. R. 1993. A generalized onehit model for bioassays of insect viruses. Biometrics 49:1136-1141.

20. Sacristán, S., Malpica, J. M., Fraile, A., and García-Arenal, F. 2003. Estimation of population bottlenecks during systemic movement of
Tobacco mosaic virus in tobacco plants. J. Virol. 77:9906-9911.

21. Smith, I. R. L., and Crook, N. E. 1988. In vivo isolation of baculovirus genotypes. Virology 166:240-244.

22. Teunis, P. F. M., Moe, C. L., Liu, P., Miller, S. E., Lindesmith, L., Baric, R. S., Le Pendu, J., and Calderon, R. L. 2008. Norwalk virus: How infectious is it? J. Med. Virol. 80:1468-1476.

23. Tromas, N., Zwart, M. P., Lafforgue, G., and Elena, S. F. 2014. Withinhost spatiotemporal dynamics of plant virus infection at the cellular level. PLoS Genet. 10:e1004186.

24. van der Werf, W., Hemerik, L., Vlak, J. M., and Zwart, M. P. 2011. Heterogeneous host susceptibility enhances prevalence of mixed-genotype micro-parasite infections. PLoS Comput. Biol. 7:e1002097.

25. Zwart, M. P., Daròs, J. A., and Elena, S. F. 2011. One is enough: In vivo effective population size is dose-dependent for a plant RNA virus. PLoS Pathog. 7:e1002122.

26. Zwart, M. P., Daròs, J. A., and Elena, S. F. 2012. Effects of potyvirus effective population size in inoculated leaves on viral accumulation and the onset of symptoms. J. Virol. 86:9737-9747.

27. Zwart, M. P., Hemerik, L., Cory, J. S., de Visser, J. A. G. M., Bianchi, F. J. J. A., van Oers, M. M., Vlak, J. M., Hoekstra, R. F., and van der Werf, W. 2009. An experimental test of the independent action hypothesis in virusinsect pathosystems. Proc. R. Soc. B 276:2233-2242.

28. Zwart, M. P., Willemsen, A., Daròs, J. A., and Elena, S. F. 2014. Experimental evolution of pseudogenization and gene loss in viral genomes. Mol. Biol. Evol. 31:121-134. 